\title{
钯催化溴代酚的 $[2+2+1]$ 去芳构螺环化反应
}

\author{
李锟雨 ${ }^{\dagger}$ 白璐 ${ }^{\dagger} *$ 奕新军* \\ (西北大学化学与材料科学学院 西安 710127)
}

\begin{abstract}
摘要 在零价钯的催化作用下, 以简单且商品化的溴代苯酚为研究目标, 炔烃作为偶联片段, 通过 $[2+2+1] 反$ 应策略, 实现了苯酚的去芳构螺环化转化, 合成了一系列新颖的含有季碳中心的螺环骨架化合物. 该方法采用的底物简单易得, 具有宽泛的底物范围, 并且可以获得较优的收率; 在非对称炔烃方面, 能以优异的区域选择性 $(>19: 1 \mathrm{rr})$ 实现这一转 化, 极大地拓宽了苯酚去芳构化的研究范围.

关键词 过渡金属催化; 去芳构化; 螺环化; 澳代酚
\end{abstract}

\section{Pd-Catalyzed Dearomative Spirocyclization of Bromophenols via $[2+2+1]$ Strategy}

\author{
Li, Kunyu ${ }^{\dagger} \quad$ Bai, Lu ${ }^{\dagger, *} \quad$ Luan, Xinjun* \\ (College of Chemistry \& Materials Science, Northwest University, Xi'an 710127)
}

\begin{abstract}
A novel palladium(0)-catalyzed dearomative spirocyclization reaction of bromophenols has been developed for building a series of spirocyclic architectures containing a quaternary carbon center via $[2+2+1]$ strategy. This method employs inexpensive bromophenols and easily accessible alkynes. It exhibits a broad substrate scope in good yields. Notably, this transformation can be realized with high regioselectivity $(>19: 1 \mathrm{rr})$ when using unsymmetrical alkynes, which greatly expands the research scope of phenol dearomatization.

Keywords transition-metal-catalyzed; dearomatization; spirocyclization; bromophenols
\end{abstract}

螺环骨架普遍存在于天然产物与药物分子中，是许 多具有药理活性分子的核心骨架 ${ }^{[1]}$. 过渡金属催化芳香 类化合物的去芳构化反应可以打破相对稳定的芳香体 系, 从平面结构转向三维立体结构, 实现螺环骨架的成 功构筑 ${ }^{[2]}$. 众所周知, 酚类化合物是一类重要的化学化 工原料 ${ }^{[3]}$, 来源极其丰富, 其衍生化转化的研究备受关 注 $^{[4]}$. 近年来, 一种新颖的转化模式一一过渡金属催化 酚类化合物的去芳构化进入了人们的视线, 并且取得了 一定的研究成果 ${ }^{[5]}$. 例如 Hamada 课题组 ${ }^{[6]}$ 、Buchwald 课题组 ${ }^{[7]}$ 以及 You 课题组 ${ }^{[8]}$ 都相继报道了酚类化合物分 子内的去芳构化反应, 构筑了一系列结构多样的螺环骨 架, 虽然该类反应官能团兼容性好, 但是其原料合成复 杂, 极大地限制了酚类化合物的去芳构化研究进展. 针

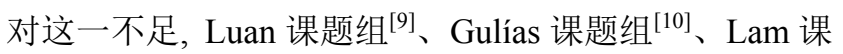

(a) Previous work ${ }^{[14]}$<smiles>[X]C1=CC=C(O)[R]=[Pt]1</smiles><smiles>[R]C#CCC#C[R]</smiles>

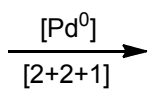<smiles>[X]CCC1=C([R])C2(C=CC(=O)C2[R])C([R])=C1C[X]</smiles>

(b) This work<smiles>[X]C1#[R]=C(O)C=C1</smiles><smiles>[R]C#[R]C#[Y]</smiles>

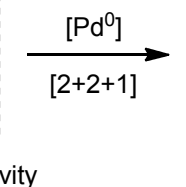

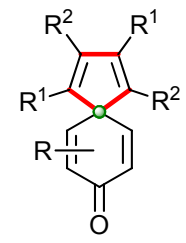

$\checkmark$ high regioselectivity

图 1 卤代酚类化合物的 $[2+2+1]$ 去芳构螺环化反应

Figure 1 Dearomatizing $[2+2+1]$ spirocyclization of halogenated phenols

\footnotetext{
* Corresponding authors. E-mail: xluan@nwu.edu.cn; bailu@nwu.edu.cn

Received March 28, 2019; revised April 26, 2019; published online May 28, 2019.

Dedicated to the 100th anniversary of the birth of Professor Ruyu Chen.

Project supported by the National Natural Science Foundation of China (No. 21672169), and the Key Laboratory Project of Xi'an City (No. 201805058ZD9CG42).

国家自然科学基金(No. 21672169)、西安市有机分子工程重点实验室(No. 201805058ZD9CG42)资助项目.

†共同第一作者(The authors contributed equally to this word).
} 
题组 ${ }^{[11]}$ 以及 You 课题组 ${ }^{[12]}$ 通过大量的探索, 采用联芳烃 酚类化合物, 以炔烃或者烯烃作为偶联片段, 成功实现 了联芳烃酚类化合物分子间的 $[3+2]$ 去芳构螺环化转 化, 弥补了分子内反应的缺陷. 但是如何发展采用简单 且商品化的酚类化合物进行去芳构化转化却是该领域 的研究难点. 2014 年, Luan 课题组 ${ }^{[13]}$ 采用简单的 2-萗酚 类化合物为底物, 通过连续插入两分子炔烃, 成功实现 了菜酚的 $[2+2+1]$ 螺环化反应. 但是这一反应区域选 择性差并且底物范围窄, 酚类化合物仅局限于萘酚, 而 对于苯酚却并未涉及. 为了解决选择性以及普适性的问 题, 2016 年, Luan 课题组 ${ }^{[14]}$ 将两分子的炔烃采用偶联片 段进行组合(图 1), 形成的双炔类化后物与简单易得的 溴代酚类化合物作用, 成功制备了一系列含有螺环骨架 的多环分子, 解决了上述 $[2+2+1]$ 螺环化反应的区域 选择性问题, 并且在该反应中, 实现了最为简单的卤代 苯酚的去芳构化转化, 为后续的研究工作提供了极大的 参考意义. 本工作在之前的研究上更进一步, 直接采用 两分子炔烃与简单易得的溴代酚类化合物偶联, 省去双 炔底物的合成步骤, 并且能以高的区域选择性实现该转 化, 较之前的研究工作有很大的进步意义.

\section{1 结果与讨论}

\section{1 反应条件优化}

选取 1-溴-2-䒺酚(1a) (0.2 $\mathrm{mmol})$ 和二苯乙炔 $(\mathbf{2 a})$ (0.5 mmol)进行探究, 采用控制变量法对反应条件进行 逐步优化. 首先, 在 $5.0 \mathrm{~mol} \% \operatorname{Pd}(\mathrm{OAc})_{2} 、 12.0 \mathrm{~mol} \%$ $\mathrm{PPh}_{3} 、 2.0$ equiv. $\mathrm{K}_{2} \mathrm{CO}_{3}$ 组成的催化体系中, 选取几种在 钯催化反应中较常使用的溶剂(表 1 ), 在 $110{ }^{\circ} \mathrm{C}$ 下反应 $18 \mathrm{~h}$. 幸运的是, 当选用甲苯 $(\mathrm{PhMe})$ 为溶剂时, 该反应 可以顺利地进行得到目标化合物, 但是只有 $37 \%$ 的产 率. 为了提高反应效率，相继尝试了乙二醇二甲醚 (DME)、1,4-二氧六环、四氢呋喃 $(\mathrm{THF})$ 和乙腈 $(\mathrm{MeCN})$ 等溶剂, 但是产率都不尽人意. 然而当以 $N, N^{\prime}$ 二二甲基甲 酰胺(DMF)为溶剂时, 能以 $58 \%$ 的产率给出目标产物. 反应溶剂确定以后, 我们认为配体会对反应效果产生较 大的影响. 只有当配体和钯前体配位之后, 才会生成真 正的催化剂来启动整个反应. 因此, 在接下来的优化中, 对一系列单膦配体和双膦配体的作用进行了考察. 结果 发现，1,3-双(二苯基膦基)丙烷(DPPP)是最高效的配体， 然而其他配体像三(2-呋喃基)非对称炔烃膦(TFP)以及 双齿配体 $(2 R, 3 S)-2.2^{\prime}$-二苯膦-1.1'-联荎(BINAP)和双(二 苯基膦基)二茂铁(DPPF)则不能很好地得到目标化合物. 基于以上结果, 可以看出配体的电子效应和位阻效应对 反应都有着非常重要的影响. 接下来选取了几种常用的 无机碱来考察不同阳离子和阴离子对反应的影响. 令
表 1 反应条件的优化 ${ }^{a}$

Table 1 Optimization of the reaction conditions
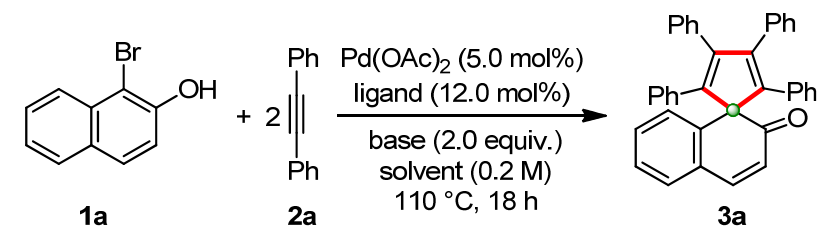

$3 a$

\begin{tabular}{|c|c|c|c|c|}
\hline Entry & Solvent & Ligand & Base & Yield $^{b} / \%$ \\
\hline 1 & Toluene & $\mathrm{PPh}_{3}$ & $\mathrm{~K}_{2} \mathrm{CO}_{3}$ & 37 \\
\hline 2 & DME & $\mathrm{PPh}_{3}$ & $\mathrm{~K}_{2} \mathrm{CO}_{3}$ & 42 \\
\hline 3 & 1,4-Dioxane & $\mathrm{PPh}_{3}$ & $\mathrm{~K}_{2} \mathrm{CO}_{3}$ & 45 \\
\hline 4 & THF & $\mathrm{PPh}_{3}$ & $\mathrm{~K}_{2} \mathrm{CO}_{3}$ & 44 \\
\hline 5 & $\mathrm{MeCN}$ & $\mathrm{PPh}_{3}$ & $\mathrm{~K}_{2} \mathrm{CO}_{3}$ & 23 \\
\hline 6 & $\mathrm{DMF}$ & $\mathrm{PPh}_{3}$ & $\mathrm{~K}_{2} \mathrm{CO}_{3}$ & 58 \\
\hline 7 & $\mathrm{DMF}$ & $\mathrm{P}\left(p-\mathrm{MeO}-\mathrm{C}_{6} \mathrm{H}_{4}\right)_{3}$ & $\mathrm{~K}_{2} \mathrm{CO}_{3}$ & 25 \\
\hline 8 & DMF & $\mathrm{P}\left(p-\mathrm{F}-\mathrm{C}_{6} \mathrm{H}_{4}\right)_{3}$ & $\mathrm{~K}_{2} \mathrm{CO}_{3}$ & 56 \\
\hline 9 & DMF & TFP & $\mathrm{K}_{2} \mathrm{CO}_{3}$ & 33 \\
\hline $10^{c}$ & $\mathrm{DMF}$ & BINAP & $\mathrm{K}_{2} \mathrm{CO}_{3}$ & 86 \\
\hline $11^{c}$ & $\mathrm{DMF}$ & DPPP & $\mathrm{K}_{2} \mathrm{CO}_{3}$ & 92 \\
\hline $12^{c}$ & $\mathrm{DMF}$ & DPPF & $\mathrm{K}_{2} \mathrm{CO}_{3}$ & 18 \\
\hline $13^{c}$ & $\mathrm{DMF}$ & DPPP & $\mathrm{Cs}_{2} \mathrm{CO}_{3}$ & 68 \\
\hline $14^{c}$ & $\mathrm{DMF}$ & DPPP & KOAc & 39 \\
\hline $15^{c}$ & DMF & DPPP & $\mathrm{K}_{3} \mathrm{PO}_{4}$ & 37 \\
\hline
\end{tabular}

人失望的是, $\mathrm{Cs}_{2} \mathrm{CO}_{3} 、 \mathrm{KOAc} 、 \mathrm{~K}_{3} \mathrm{PO}_{4}$ 均使反应效率降低. 这些实验结果表明 $\mathrm{K}_{2} \mathrm{CO}_{3}$ 是最适合这类反应的碱. 基于 以上的条件优化，最终确定了最优反应条件： $5.0 \mathrm{~mol} \%$ $\mathrm{Pd}(\mathrm{OAc})_{2} 、 6.0 \mathrm{~mol} \%$ DPPP、 2.0 equiv. $\mathrm{K}_{2} \mathrm{CO}_{3} 、 \mathrm{DMF}(0.2$ $\mathrm{mol} / \mathrm{L}$ )为溶剂，在 $110{ }^{\circ} \mathrm{C}$ 下应 $18 \mathrm{~h}$.

\section{2 卤代酚类化合物的拓展}

如何实现最简单酚类化合物的去芳构化转化一直 是该领域的研究热点. 在最优反应条件下, 选用二苯乙 炔作为偶联片段，对不同酚类化合物的范围进行了考察 (表 2). 首先, 尝试了最简单的对碘苯酚(1b)的去芳构化 转化, 虽然只获得了 $40 \%$ 的产率, 但是这在简单卤代酚 的去芳构化进展中已经有了很大的进步. 随后又考察了 一系列带有各种官能团以及不同位置取代的对澳苯酚 类底物，都可以顺利进行并且取得了较高的收率. 从表 2 可以看出，当对澳苯酚带有给电子取代基 $\mathrm{Me}(1 \mathrm{c} 、 1 d$ 、 1e、1k、1l), OMe (1f), ${ }^{i} \operatorname{Pr}(\mathbf{1 m}),{ }^{t} \mathrm{Bu}(\mathbf{1 n})$ 时，反应表现良 好，并且不受官能团取代位置的影响，可以在 2、3、5、 6 位分别取代. 当取代基转变为吸电子取代基 $F(1 \mathrm{~g} 、 1 \mathbf{j})$, $\mathrm{Cl}(\mathbf{1 h}), \mathrm{CF}_{3}$ (1i) 时，也可以获得良好的收率. 尝试了对 溴荎酚 $(10)$ 的反应活性，以 $78 \%$ 的收率顺利进 
表 2 对溴代酚的拓展 ${ }^{a}$

Table 2 Scope of para-bromophenols

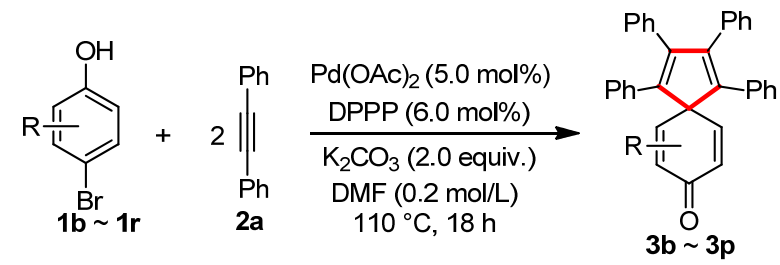

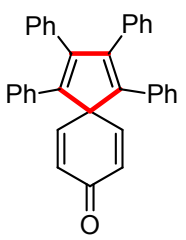

3b $46 \%^{b}$

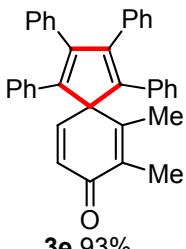

3e $93 \%$

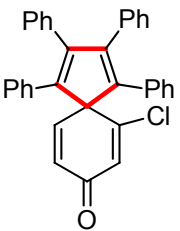

3h $48 \%$

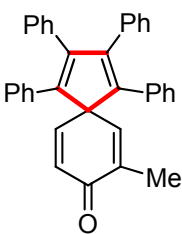

3k $94 \%$<smiles>CC(C)(C)C1=CC2(C=C(C(C)(C)C)C(=O)C1=O)C(c1ccccc1)=C(c1ccccc1)C(c1ccccc1)=C2c1ccccc1</smiles>

3n $67 \%$

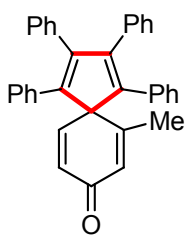

3c $92 \%$
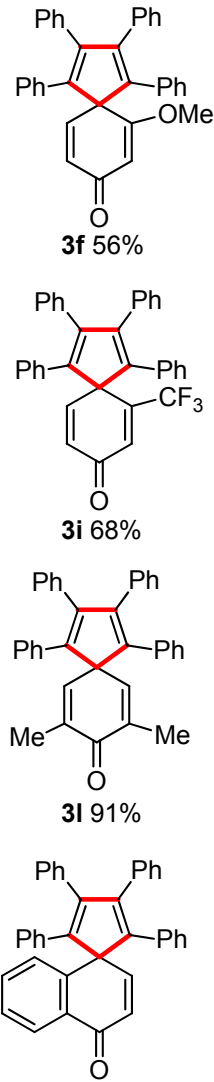

3o $78 \%$

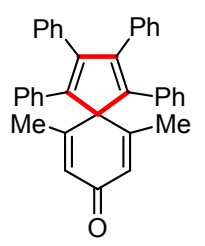

3d $90 \%$

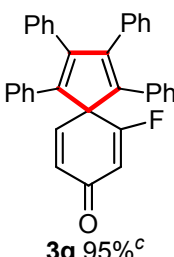<smiles>O=C1C=CC2(C=C1F)C(c1ccccc1)=C(c1ccccc1)C(c1ccccc1)=C2c1ccccc1</smiles><smiles>CC(C)C1=CC2(C=C(C(C)C)C(=O)C1=O)C(c1ccccc1)=C(c1ccccc1)C2c1ccccc1</smiles>

$3 \mathrm{~m} 78 \%$<smiles>O=C1C=CC2(C3=C1CCCC3)C(c1ccccc1)=C(c1ccccc1)C(c1ccccc1)=C2c1ccccc1</smiles>

$3 p 52 \%$
${ }^{a}$ Reaction conditions: 1a $(0.2 \mathrm{mmol}), 2 \mathrm{a}(0.5 \mathrm{mmol}) .{ }^{b}$ Reaction at $40{ }^{\circ} \mathrm{C}, 48$ h. ${ }^{c}$ Reaction at $90{ }^{\circ} \mathrm{C}$.

行. 值得一提的是, 当采用 2,3 位被取代的对溴苯酚 (1p)时，该过程不仅可以顺利进行并且能以 $52 \%$ 的收率 获得螺 [4.5]骨架. 通过对溴代苯酚底物的拓展, 发现该 反应兼容性好, 可以兼容各类取代基, 并且以良好的收 率获得了一系列螺环骨架.

\section{3 炔烃底物范围拓展}

为了验证 $[2+2+1]$ 反应的重要性和可行性, 对炔
烃的普适性进行了研究与扩展. 首先尝试了挑战性较小 的对称炔烃，如表 3 所示，芳基取代的炔烃、烷基取代 的炔烃以及杂环取代的炔烃在该反应中都能很好地兼 容. 尝试了在芳环的各个位置带上不同的取代基，包括 对位的 $\mathrm{Me}(\mathbf{2 b}) 、 \mathrm{OMe}(\mathbf{2 c}) 、 \mathrm{CF}_{3}(\mathbf{2 d}) 、 \mathrm{Cl}(\mathbf{2 e})$ 等取代基, 都可以得到优异的收率. 除对位取代外，间位取代以及 邻位取代这一类位阻较大的炔烃 $(\mathbf{2 f} 、 \mathbf{2 g})$ 也分别能以 $90 \%$ 以及 $92 \%$ 的收率完成该反应. 值得注意的是, 采用 后续衍生化可能性大的的烷基取代炔烃时，能以 $76 \%$ 的 收率获得目标化合物. 令人欣喜的是，引入噻吩骨架的 炔烃时, 虽然只能得到 $42 \%$ 的收率, 但是却极大地丰富 了该类反应的底物范围. 该反应最大的难点就是采用非 对称炔烃时实现其区域选择性的控制，所以考察了一端 带有烷基一端带有芳基的不对称炔烃, 都能以较好的收 率获得目标化合物, 这一系列的非对称炔烃都能获得优 异的区域选择性( $>19: 1 r r)$.

表 3 炔烃的拓展 ${ }^{a}$

Table 3 Scope of alkynes
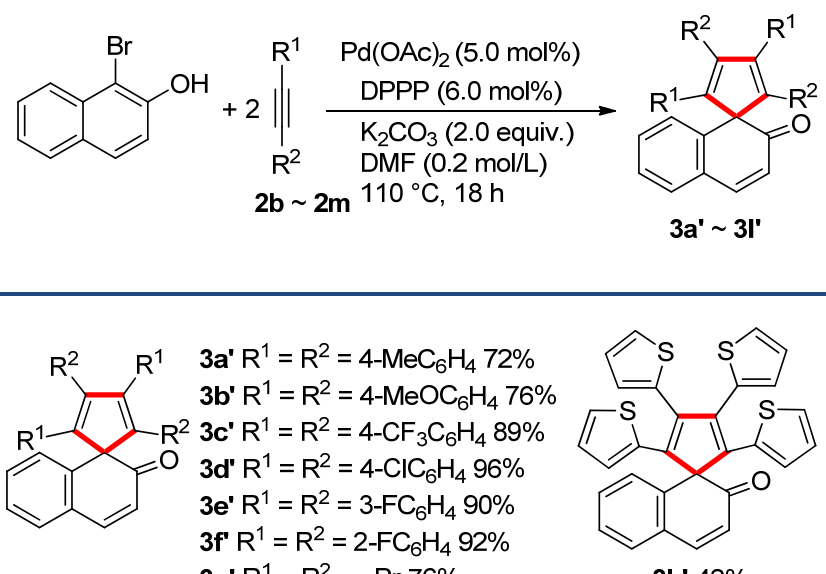

3' $\mathbf{R}^{1}=\mathrm{R}^{2}=n \operatorname{Pr} 76 \%$

$3 h^{\prime} 42 \%$

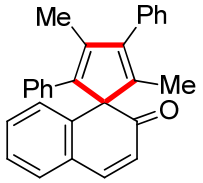

$3 \mathbf{i}^{\prime} 76 \%$

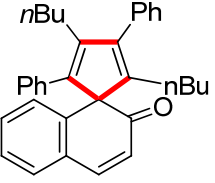

$3 j^{\prime} 54 \%$

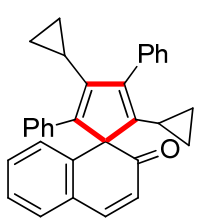

3k' $67 \%$
${ }^{a}$ Reaction conditions: 1a $(0.2 \mathrm{mmol}), \mathbf{2 a}(0.5 \mathrm{mmol})$

\section{2 结论}

以 $\operatorname{Pd}(0)$ 催化为核心手段, 瞄准商品化的溴代苯酚 类化合物，以简单炔烃作为偶联片段，通过连续插入两 分子炔烃，成功实现了 $[2+2+1]$ 去芳构螺环化转化，获 得了一系列含有季碳中心的螺环骨架化合物. 值得注意 的是，当采用非对称炔烃时，最终以高区域选择性实现 了这一 $[2+2+1]$ 螺环化反应. 


\section{3 实验部分}

\section{1 仪器与试剂}

实验所涉及到的催化反应都在手套箱(美国创新科 技公司)里进行. ${ }^{1} \mathrm{H}$ NMR $(400 \mathrm{MHz})$ 和 ${ }^{13} \mathrm{C}$ NMR (100 $\mathrm{MHz}$ )在 Varian Unity Inova-400 (400 MHz)或者 Bruker Ascend 400 (400 MHz)超导核磁共振仪上测定, 氞代试 剂是 $\mathrm{CDCl}_{3}$. IR 在 BRUKER TENSOR 27 型红外光谱分 析仪上测定. HRMS 在 Daltonics MicroTof-Q II 型液质联 用电喷雾四级杆飞行时间串联质谱仪上测定.

\section{2 实验方法}

在 $5 \mathrm{~mL}$ 的微波反应瓶中依次加入澳代酚类底物 $(0.2 \mathrm{mmol})$ 、炔烃底物 $(0.5 \mathrm{mmol}) 、 \mathrm{Pd}(\mathrm{OAc})_{2}(5.0$ $\mathrm{mmol} \%) 、 \operatorname{DPPP}(6.0 \mathrm{mmol} \%)$ 和 $\mathrm{K}_{2} \mathrm{CO}_{3}$ (2.0 equiv.), 再使 用移液枪向反应瓶中加入 $1.0 \mathrm{~mL}$ DMF 溶剂, 在 $110{ }^{\circ} \mathrm{C}$ 下反应 $18 \mathrm{~h}$. 冷至室温后将反应液用一定量的氯化铵饱 和溶液和乙酸乙酯萃取, 萃取操作进行 $2 \sim 3$ 次. 萃取所 得的有机混合液经 $\mathrm{MgSO}_{4}$ 干燥后, 利用旋转蒸发仪将 溶剂和乙酸乙酯除去, 再利用柱层析 $[V$ (石油醚) $: V($ 乙 酸乙酯) $=20: 1$ 分离粗产物, 得到纯的目标产物.

2,3,4,5-四苯基-2' $H$-螺 [环戊烷-1,1'-菜]-2,4-二烯-2'酮(3a) ${ }^{[13]}$ : 黄色油状物, 产率 $92 \% .{ }^{1} \mathrm{H}$ NMR $(400 \mathrm{MHz}$, $\mathrm{CDCl} 3) \delta: 7.31 \sim 7.23(\mathrm{~m}, 2 \mathrm{H}), 7.20 \sim 7.16(\mathrm{~m}, 2 \mathrm{H})$, $7.06 \sim 6.98(\mathrm{~m}, 6 \mathrm{H}), 6.96 \sim 6.88(\mathrm{~m}, 6 \mathrm{H}), 6.88 \sim 6.82(\mathrm{~m}$, $4 \mathrm{H}), 6.57(\mathrm{~d}, J=7.6 \mathrm{~Hz}, 4 \mathrm{H}), 6.10(\mathrm{~d}, J=9.9 \mathrm{~Hz}, 1 \mathrm{H})$.

1,2,3,4-四苯基螺 [4.5]癸-1,3,6,9-四烯-8-酮(3b): 黄 色油状物, 产率 $46 \% .{ }^{1} \mathrm{H}$ NMR $\left(400 \mathrm{MHz}, \mathrm{CDCl}_{3}\right) \delta:{ }^{1} \mathrm{H}$ NMR (400 MHz, $\left.\mathrm{CDCl}_{3}\right) \delta: 7.15 \sim 7.07(\mathrm{~m}, 12 \mathrm{H}), 7.07 \sim$ $7.04(\mathrm{~m}, 4 \mathrm{H}), 6.90$ (d, $J=6.9 \mathrm{~Hz}, 4 \mathrm{H}), 6.82(\mathrm{~d}, J=9.9 \mathrm{~Hz}$, $2 \mathrm{H}), 6.44(\mathrm{~d}, J=9.9 \mathrm{~Hz}, 2 \mathrm{H}) ;{ }^{13} \mathrm{C}$ NMR $\left(100 \mathrm{MHz}, \mathrm{CDCl}_{3}\right)$ $\delta: 186.2,147.9,147.6,141.2,134.6,134.5,131.8,129.9$, $129.3,128.0,127.9,127.5,127.3,66.2$; IR (KBr) v: 2922, 2853,1651, 1444, 1169, 1074, 864, $739 \mathrm{~cm}^{-1}$. HRMS (ESI) calcld for $\mathrm{C}_{24} \mathrm{H}_{24} \mathrm{ONa}[\mathrm{M}+\mathrm{Na}]^{+} 471.1725$, found 471.1747.

6-甲基-1,2,3,4-四苯基螺 [4.5]癸-1,3,6,9-四烯-8-酮 (3c): 黄色油状物, 产率 $92 \% .{ }^{1} \mathrm{H}$ NMR $(400 \mathrm{MHz}$, $\left.\mathrm{CDCl}_{3}\right) \delta: 7.20 \sim 7.12(\mathrm{~m}, 12 \mathrm{H}), 7.08(\mathrm{~s}, 4 \mathrm{H}), 6.98(\mathrm{~d}, J=$ $7.0 \mathrm{~Hz}, 4 \mathrm{H}), 6.82(\mathrm{~d}, J=9.7 \mathrm{~Hz}, 1 \mathrm{H}), 6.49(\mathrm{~d}, J=9.7 \mathrm{~Hz}$, $1 \mathrm{H}), 6.39(\mathrm{~s}, 1 \mathrm{H}), 1.97(\mathrm{~s}, 3 \mathrm{H}) ;{ }^{13} \mathrm{C}$ NMR $(100 \mathrm{MHz}$, $\left.\mathrm{CDCl}_{3}\right) \delta: 187.0,156.7,148.6,148.4,142.1,134.8,134.2$, $131.3,130.9,129.9,129.0,128.1,128.0,127.5,127.4$, 68.7, 19.0; IR (KBr) v: 2923, 2854, 1664, 1446, 1172, $1075,835,745 \mathrm{~cm}^{-1}$. HRMS (ESI) calcld for $\mathrm{C}_{35} \mathrm{H}_{26} \mathrm{ONa}$ $[\mathrm{M}+\mathrm{Na}]^{+}$485.1881, found 485.1882.
6,10-二甲基-1,2,3,4-四苯基螺[4.5]癸-1,3,6,9-四烯8-酮(3d)：白色固体，产率 90\%. m.p. $223 \sim 226{ }^{\circ} \mathrm{C} ;{ }^{1} \mathrm{H}$ NMR (400 MHz, $\left.\mathrm{CDCl}_{3}\right) \delta: 7.20 \sim 7.10(\mathrm{~m}, 12 \mathrm{H}), 7.00 \sim$ $6.96(\mathrm{~m}, 4 \mathrm{H}), 6.96 \sim 6.92(\mathrm{~m}, 4 \mathrm{H}), 6.35(\mathrm{~s}, 2 \mathrm{H}), 1.96(\mathrm{~s}$, $6 \mathrm{H}) ;{ }^{13} \mathrm{C}$ NMR $\left(100 \mathrm{MHz}, \mathrm{CDCl}_{3}\right) \delta: 186.6,156.7,149.2$, $143.2,134.9,133.8,130.8,129.8,128.7,128.1,128.0$, 127.5, 127.4, 71.2, 18.4; IR (KBr) v: 3055, 2854, 1660, $1441,1157,1074,889,740 \mathrm{~cm}^{-1}$. HRMS (ESI) calcld for $\mathrm{C}_{36} \mathrm{H}_{28} \mathrm{ONa}[\mathrm{M}+\mathrm{Na}]^{+}$499.2038, found 499.2041.

6,7-二甲基-1,2,3,4-四苯基螺 [4.5]癸-1,3,6,9-四烯-8酮(3e): 黄色油状物，产率 93\%. ${ }^{1} \mathrm{H}$ NMR $(400 \mathrm{MHz}$, $\left.\mathrm{CDCl}_{3}\right) \delta: 7.19 \sim 7.10(\mathrm{~m}, 12 \mathrm{H}), 7.03 \sim 6.99(\mathrm{~m}, 4 \mathrm{H})$, $6.98 \sim 6.94(\mathrm{~m}, 4 \mathrm{H}), 6.75(\mathrm{~d}, J=9.7 \mathrm{~Hz}, 1 \mathrm{H}), 6.49(\mathrm{~d}, J=$ $9.7 \mathrm{~Hz}, 1 \mathrm{H}), 1.89(\mathrm{~d}, J=13.4 \mathrm{~Hz}, 6 \mathrm{H}) ;{ }^{13} \mathrm{C}$ NMR $(100$ $\left.\mathrm{MHz}, \mathrm{CDCl}_{3}\right) \delta: 186.3,149.0,148.2,147.2,142.6,136.0$, $135.0,134.4,130.5,129.9,129.0,128.0,127.4,127.3$, 69.1, 16.1, 11.2; IR (KBr) v: 3055, 2923, 2856, 1882, 1806, 1073, 833, $745 \mathrm{~cm}^{-1}$. HRMS (ESI) calcld for $\mathrm{C}_{36} \mathrm{H}_{29} \mathrm{O}[\mathrm{M}+\mathrm{H}]^{+}$477.2218, found 477.2220.

6-甲氧基-1,2,3,4-四苯基螺[4.5]癸-1,3,6,9-四烯-8-酮 (3f): 无色油状物, 产率 $56 \% .{ }^{1} \mathrm{H}$ NMR (400 MHz, $\left.\mathrm{CDCl}_{3}\right) \delta: 7.29 \sim 7.21(\mathrm{~m}, 12 \mathrm{H}), 7.15 \sim 7.10(\mathrm{~m}, 4 \mathrm{H})$, $7.08 \sim 7.03(\mathrm{~m}, 4 \mathrm{H}), 6.62(\mathrm{~d}, J=9.7 \mathrm{~Hz}, 1 \mathrm{H}), 6.48(\mathrm{~d}, J=$ $9.6 \mathrm{~Hz}, 1 \mathrm{H}), 5.84(\mathrm{~s}, 1 \mathrm{H}), 3.79(\mathrm{~s}, 3 \mathrm{H}) ;{ }^{13} \mathrm{C}$ NMR $(100$ $\left.\mathrm{MHz}, \mathrm{CDCl}_{3}\right) \delta: 188.8,172.8,147.6,144.1,142.4,134.7$, $134.3,130.1,130.0,129.1,127.9,127.7,127.4,127.1$, 67.5, 55.7; IR (KBr) v: 3056, 2925, 2852, 1665, 1623, 1447, 1235, 842, $736 \mathrm{~cm}^{-1}$. HRMS (ESI) calcld for $\mathrm{C}_{35} \mathrm{H}_{26} \mathrm{O}_{2} \mathrm{Na}[\mathrm{M}+\mathrm{Na}]^{+}$501.1830, found 501.1821.

6-氟-1,2,3,4-四苯基螺[4.5]癸-1,3,6,9-四烯-8-酮(3g): 黄色固体, 产率 $95 \%$. m.p. $124 \sim 126{ }^{\circ} \mathrm{C} ;{ }^{1} \mathrm{H}$ NMR (400 $\left.\mathrm{MHz}, \mathrm{CDCl}_{3}\right) \delta: 7.21 \sim 7.11(\mathrm{~m}, 16 \mathrm{H}), 6.93(\mathrm{~d}, J=7.1 \mathrm{~Hz}$, $4 \mathrm{H}), 6.64(\mathrm{t}, J=10.2 \mathrm{~Hz}, 1 \mathrm{H}), 6.40(\mathrm{~d}, J=9.6 \mathrm{~Hz}, 1 \mathrm{H})$, $6.10(\mathrm{~d}, J=13.9 \mathrm{~Hz}, 1 \mathrm{H}) ;{ }^{13} \mathrm{C}$ NMR $\left(100 \mathrm{MHz}, \mathrm{CDCl}_{3}\right) \delta$ : 185.3, 150.5, 149.2, 139.5, 134.2 (d, $J=95.6 \mathrm{~Hz}), 129.8$, 129.7, 128.0, 128.0, 127.6 (d, $J=19.6 \mathrm{~Hz}), 64.6$; IR (KBr) $v: 3054,2923,2853,1667,1616,1442,1260,874,798$ $\mathrm{cm}^{-1}$. HRMS (ESI) calcld for $\mathrm{C}_{34} \mathrm{H}_{23} \mathrm{FONa}[\mathrm{M}+\mathrm{Na}]^{+}$ 489.1631, found 489.1631 .

6-氯-1,2,3,4-四苯基螺[4.5]癸-1,3,6,9-四烯-8-酮(3h): 白色固体, 产率 $48 \%$. m.p. $218 \sim 219{ }^{\circ} \mathrm{C} ;{ }^{1} \mathrm{H}$ NMR (400 $\left.\mathrm{MHz}, \mathrm{CDCl}_{3}\right) \delta: 7.23 \sim 7.11(\mathrm{~m}, 12 \mathrm{H}), 7.11 \sim 7.07(\mathrm{~m}, 4 \mathrm{H})$, $6.94(\mathrm{~d}, J=7.0 \mathrm{~Hz}, 4 \mathrm{H}), 6.84$ (d, $J=9.7 \mathrm{~Hz}, 1 \mathrm{H}), 6.60$ (s, $1 \mathrm{H}), 6.46(\mathrm{~d}, J=9.7 \mathrm{~Hz}, 1 \mathrm{H}) ;{ }^{13} \mathrm{C} \mathrm{NMR}\left(100 \mathrm{MHz}, \mathrm{CDCl}_{3}\right)$ $\delta: 185.5,153.5,149.3,147.5,141.0,134.5,133.8,132.5$, 
130.5, 130.0, 129.4, 128.2, 128.0, 127.9, 127.6, 69.9; IR (KBr) v: 3055, 2923, 2853, 1652, 1613, 1440, 1266, 884, $739 \mathrm{~cm}^{-1}$. HRMS (ESI) calcld for $\mathrm{C}_{34} \mathrm{H}_{23} \mathrm{ClONa}[\mathrm{M}+$ $\mathrm{Na}]^{+}$505.1335, found 505.1331.

6-三氟甲基-1,2,3,4-四苯基螺[4.5]癸-1,3,6,9-四烯-8酩(3i): 黄色油状物, 产率 $68 \% .{ }^{1} \mathrm{H}$ NMR $(400 \mathrm{MHz}$, $\left.\mathrm{CDCl}_{3}\right) \delta: 7.17 \sim 7.10(\mathrm{~m}, 12 \mathrm{H}), 7.06(\mathrm{~d}, J=6.7 \mathrm{~Hz}, 4 \mathrm{H})$, $6.92(\mathrm{~d}, J=6.8 \mathrm{~Hz}, 4 \mathrm{H}), 6.87(\mathrm{~s}, 1 \mathrm{H}), 6.75(\mathrm{~d}, J=9.8 \mathrm{~Hz}$, $1 \mathrm{H}), 6.51(\mathrm{~d}, J=9.8 \mathrm{~Hz}, 1 \mathrm{H}) ;{ }^{13} \mathrm{C} \mathrm{NMR}\left(100 \mathrm{MHz}, \mathrm{CDCl}_{3}\right)$ $\delta: 188.7$ (q, $J=15.7 \mathrm{~Hz}), 175.3,172.5,149.1,144.0$ (q, $J=$ $5.6 \mathrm{~Hz}), 140.0,134.3,133.8,130.0,129.3,128.3,128.0$, 127.9, 127.6, 113.5 (q, $J=10.1 \mathrm{~Hz}), 100.1,66.9$ (q, $J=$ $24.3 \mathrm{~Hz}$ ); IR (KBr) v: 3060, 2923, 2854, 1664, 1617, 1446, $1265,835,745 \mathrm{~cm}^{-1}$. HRMS (ESI) calcld for $\mathrm{C}_{35} \mathrm{H}_{25} \mathrm{~F}_{3} \mathrm{O}$ $[\mathrm{M}+\mathrm{H}]^{+}$517.1779, found 517.1797.

7-氟-1,2,3,4-四苯基螺[4.5]癸-1,3,6,9-四烯-8-酮(3j): 白色固体, 产率 54\%. m.p. $210 \sim 212{ }^{\circ} \mathrm{C} ;{ }^{1} \mathrm{H}$ NMR (400 $\left.\mathrm{MHz}, \mathrm{CDCl}_{3}\right) \delta: 7.21 \sim 7.11(\mathrm{~m}, 16 \mathrm{H}), 6.93(\mathrm{~d}, J=7.1 \mathrm{~Hz}$, 4H), 6.64 (t, $J=10.2 \mathrm{~Hz}, 1 \mathrm{H}), 6.40(\mathrm{~d}, J=9.6 \mathrm{~Hz}, 1 \mathrm{H})$, $6.10(\mathrm{~d}, J=13.9 \mathrm{~Hz}, 1 \mathrm{H}),{ }^{13} \mathrm{C}$ NMR $\left(100 \mathrm{MHz}, \mathrm{CDCl}_{3}\right) \delta$ : $178.8(\mathrm{~d}, J=21.2 \mathrm{~Hz}), 156.5$ (q, $J=264.5 \mathrm{~Hz}), 149.0$, 147.7, 140.7, 134.5, 134.3, 131.6 (q, $J=4.5 \mathrm{~Hz}), 130.0$, $129.4,128.3,128.0,127.9,127.5,123.3$ (q, $J=14.5 \mathrm{~Hz}$ ), 100.1, 66.6. IR (KBr) v: 3056, 2920, 2852, 1662, 1613, $1441,1258,873,795 \mathrm{~cm}^{-1}$. HRMS (ESI) calcld for $\mathrm{C}_{34} \mathrm{H}_{23} \mathrm{FONa}[\mathrm{M}+\mathrm{Na}]^{+}$489.1631, found 489.1631.

7- 甲基-1,2,3,4-四苯基螺 [4.5]癸-1,3,6,9-四烯-8-酮 (3k): 黄色油状物, 产率 94\%. ${ }^{1} \mathrm{H}$ NMR $(400 \mathrm{MHz}$, $\left.\mathrm{CDCl}_{3}\right) \delta: 7.21 \sim 7.05(\mathrm{~m}, 16 \mathrm{H}), 6.95(\mathrm{~d}, J=6.8 \mathrm{~Hz}, 4 \mathrm{H})$, $6.97 \sim 6.93(\mathrm{~m}, 1 \mathrm{H}), 6.66(\mathrm{~s}, 1 \mathrm{H}), 6.46(\mathrm{~d}, J=9.7 \mathrm{~Hz}, 1 \mathrm{H})$, 1.93 (s, 3H); ${ }^{13} \mathrm{C}$ NMR $\left(100 \mathrm{MHz}, \mathrm{CDCl}_{3}\right) \delta: 186.8,147.7$, $147.2,142.7,141.6,138.5,134.9,134.7,131.5,130.0$, 129.3, 127.9, 127.8, 127.4, 127.2, 66.6, 16.1; IR (KBr) v: 2930, 2848, 1659, 1452, 1183, 1058, 866, $796 \mathrm{~cm}^{-1}$. HRMS (ES) calcld for $\mathrm{C}_{35} \mathrm{H}_{26} \mathrm{ONa}[\mathrm{M}+\mathrm{Na}]^{+}$485.1881, found 485.1881 .

7, 9-二甲基-1,2,3,4-四苯基螺[4.5]癸-1,3,6,9-四烯-8酮(3I): 黄色油状物, 产率 91\%. ${ }^{1} \mathrm{H}$ NMR (400 MHz, $\left.\mathrm{CDCl}_{3}\right) \delta: 7.08 \sim 6.98(\mathrm{~m}, 12 \mathrm{H}), 6.95 \sim 6.90(\mathrm{~m}, 4 \mathrm{H})$, $6.84 \sim 6.79(\mathrm{~m}, 4 \mathrm{H}), 6.49(\mathrm{~s}, 2 \mathrm{H}), 1.79(\mathrm{~s}, 6 \mathrm{H}) ;{ }^{13} \mathrm{C} \mathrm{NMR}$ $\left(100 \mathrm{MHz}, \mathrm{CDCl}_{3}\right) \delta: 187.4,146.7,142.4,142.1,137.9$, $135.2,135.0,130.1,129.4,127.9,127.9,127.3,127.2$, 66.3, 16.4; IR (KBr) v: 3055, 2852, 1658, 1441, 1211, $1063,910,742 \mathrm{~cm}^{-1}$. HRMS (ESI) calcld for $\mathrm{C}_{36} \mathrm{H}_{29} \mathrm{O}$ $[\mathrm{M}+\mathrm{H}]^{+}$477.2218, found 477.2215.
7，9-二异丙基-1,2,3,4-四苯基螺 [4.5]癸-1,3,6,9-四 烯-8-酮(3m): 黄色油状物, 产率 78\%. ${ }^{1} \mathrm{H}$ NMR (400 $\left.\mathrm{MHz}, \mathrm{CDCl}_{3}\right) \delta: 7.16 \sim 7.06(\mathrm{~m}, 12 \mathrm{H}), 7.03 \sim 6.98(\mathrm{~m}$, $4 \mathrm{H}), 6.98 \sim 6.93(\mathrm{~m}, 4 \mathrm{H}), 6.44(\mathrm{~s}, 2 \mathrm{H}), 3.05 \sim 2.90(\mathrm{~m}$, $2 \mathrm{H}), 0.95(\mathrm{~d}, J=6.9 \mathrm{~Hz}, 12 \mathrm{H}) ;{ }^{13} \mathrm{C}$ NMR $(100 \mathrm{MHz}$, $\left.\mathrm{CDCl}_{3}\right) \delta: 185.3,147.7,146.3,142.7,139.0,135.2,131.4$, 130.1, 129.4, 127.9, 127.8, 127.2, 127.1, 65.5, 26.6, 21.9. IR (KBr) v: 3056, 2926, 1652, 1441, 1136, 1071, 797, 745 $\mathrm{cm}^{-1}$. HRMS (ESI) calcld for $\mathrm{C}_{40} \mathrm{H}_{36} \mathrm{ONa}[\mathrm{M}+\mathrm{Na}]^{+}$ 555.2664, found 555.2665.

7，9-二叔丁基-1,2,3,4-四苯基螺 [4.5]癸-1,3,6,9-四 烯-8-酮(3n): 黄色油状物, 产率 67\%。 ${ }^{1} \mathrm{H}$ NMR (400 $\left.\mathrm{MHz}, \mathrm{CDCl}_{3}\right) \delta: 7.16 \sim 7.06(\mathrm{~m}, 12 \mathrm{H}), 7.03 \sim 6.95(\mathrm{~m}$, $8 \mathrm{H}), 6.40$ (s, 2H), 1.11 (s, 18H); ${ }^{13} \mathrm{C}$ NMR $(100 \mathrm{MHz}$, $\left.\mathrm{CDCl}_{3}\right) \delta: 186.2,149.4,145.9,143.0,138.5,135.1,135.0$, 130.1, 129.3, 127.8, 127.6, 127.1, 65.4, 35.2, 29.3; IR (KBr) v: 3055, 2933, 1652, 1442, 1122, 1072, 807, 742 $\mathrm{cm}^{-1}$. HRMS (ESI) calcld for $\mathrm{C}_{42} \mathrm{H}_{40} \mathrm{ONa}[\mathrm{M}+\mathrm{Na}]^{+}$ 583.2977, found 583.2968.

2,3,4,5-四苯基氢-螺[环戊烷-1,1'-芸]-2,4-二烯-4'-酮 (3o): 黄色固体, 产率 $78 \%$. m.p. 86 87 ${ }^{\circ} \mathrm{C} ;{ }^{1} \mathrm{H}$ NMR $\left(400 \mathrm{MHz}, \mathrm{CDCl}_{3}\right) \delta: 8.01$ (d, $\left.J=7.5 \mathrm{~Hz}, 1 \mathrm{H}\right), 7.44 \sim 7.39$ (m, $1 \mathrm{H}), 7.33 \sim 7.27(\mathrm{~m}, 2 \mathrm{H}), 7.10 \sim 7.01(\mathrm{~m}, 6 \mathrm{H}), 6.97 \sim$ $6.83(\mathrm{~m}, 10 \mathrm{H}), 6.82 \sim 6.78(\mathrm{~m}, 1 \mathrm{H}), 6.69 \sim 6.61(\mathrm{~m}, 4 \mathrm{H})$, $6.50(\mathrm{~d}, J=9.9 \mathrm{~Hz}, 1 \mathrm{H}) ;{ }^{13} \mathrm{C} \mathrm{NMR}\left(100 \mathrm{MHz}, \mathrm{CDCl}_{3}\right) \delta$ : $185.3,148.8,146.9,145.7,140.1,135.0,134.5,133.2$, $133.0,130.7,130.1,129.3,128.0,127.9,127.2,126.5$, 66.3; IR (KBr) v: 3041, 2925, 2855, 1665, 1425, 1261, $874,742 \mathrm{~cm}^{-1}$. HRMS (ESI) calcld for $\mathrm{C}_{38} \mathrm{H}_{26} \mathrm{ONa}$ $[\mathrm{M}+\mathrm{Na}]^{+}$521.1881, found 521.1872.

2,3,4,5-四苯基-5',6', $7^{\prime}, 8^{\prime}$ - 四氢-4' $H$ 螺 [环戊烷-1,1'菜]-2,4-二烯-4'-酮 (3p): 黄色固体, 产率 52\%. m.p. $165 \sim 167{ }^{\circ} \mathrm{C} ;{ }^{1} \mathrm{H}$ NMR $\left(400 \mathrm{MHz}, \mathrm{CDCl}_{3}\right) \delta: 7.18 \sim 7.08$ (m, $6.2 \mathrm{~Hz}, 12 \mathrm{H}), 7.01$ (d, $J=6.3 \mathrm{~Hz}, 4 \mathrm{H}), 6.94$ (d, $J=6.7$ $\mathrm{Hz}, 4 \mathrm{H}), 6.72(\mathrm{~d}, J=9.7 \mathrm{~Hz}, 1 \mathrm{H}), 6.46(\mathrm{~d}, J=9.7 \mathrm{~Hz}, 1 \mathrm{H})$, $2.35(\mathrm{~s}, 2 \mathrm{H}), 2.21(\mathrm{~s}, 2 \mathrm{H}), 1.61(\mathrm{~d}, J=7.0 \mathrm{~Hz}, 4 \mathrm{H}) ;{ }^{13} \mathrm{C}$ NMR $\left(100 \mathrm{MHz}, \mathrm{CDCl}_{3}\right) \delta: 186.6,151.3,148.1,147.8$, $142.9,136.9,135.2,134.4,130.7,130.0,129.1,128.1$, 128.0, 127.4, 127.3, 68.5, 26.0, 22.7, 22.1, 22.0; IR (KBr) v: 3054, 2876, 1672, 1630, 1435, 1292, 863, $774 \mathrm{~cm}^{-1}$. HRMS (ESI) calcld for $\mathrm{C}_{38} \mathrm{H}_{30} \mathrm{ONa}[\mathrm{M}+\mathrm{Na}]^{+}$525.2194, found 525.2180 .

2,3,4,5-四-对-甲苯基-2' $H$-螺 [环戊二烯并 [2,4]二烯1,1'-萗]-2'-酮 (3a') ${ }^{[13]}$ : 黄色固体, 产率 $72 \%$. m.p. 203 $204{ }^{\circ} \mathrm{C} ;{ }^{1} \mathrm{H}$ NMR $\left(400 \mathrm{MHz}, \mathrm{CDCl}_{3}\right) \delta: 7.37 \sim 7.32$ (m, 
$2 \mathrm{H}), 7.31 \sim 7.28(\mathrm{~m}, 2 \mathrm{H}), 7.27(\mathrm{~s}, 1 \mathrm{H}), 6.91 \sim 6.83(\mathrm{~m}$, $8 \mathrm{H}), 6.75$ (d, $J=7.9 \mathrm{~Hz}, 4 \mathrm{H}), 6.54$ (d, $J=8.1 \mathrm{~Hz}, 4 \mathrm{H}), 6.20$ $(\mathrm{d}, J=9.9 \mathrm{~Hz}, 1 \mathrm{H}), 2.28(\mathrm{~s}, 6 \mathrm{H}), 2.16(\mathrm{~s}, 6 \mathrm{H})$.

$2,3,4,5$-四-对-甲氧基苯基-2' $H$-螺 [环戊二烯并 $[2,4]$ 二烯-1,1'-菜]-2'-酮 $\left(3 \mathbf{b}^{\prime}\right)^{[13]}$ : 黄色固体, 产率 76\%. m.p. $200 \sim 201{ }^{\circ} \mathrm{C} ;{ }^{1} \mathrm{H}$ NMR (400 MHz, $\left.\mathrm{CDCl}_{3}\right) \delta: 7.35 \sim 7.30$ $(\mathrm{m}, 3 \mathrm{H}), 7.27 \sim 7.24(\mathrm{~m}, 2 \mathrm{H}), 6.91(\mathrm{~d}, J=8.5 \mathrm{~Hz}, 4 \mathrm{H})$, $6.66(\mathrm{~d}, J=8.6 \mathrm{~Hz}, 4 \mathrm{H}), 6.56(\mathrm{~d}, J=8.8 \mathrm{~Hz}, 4 \mathrm{H}), 6.48$ (d, $J=8.7 \mathrm{~Hz}, 4 \mathrm{H}), 6.18(\mathrm{~d}, J=9.9 \mathrm{~Hz}, 1 \mathrm{H}), 3.75$ (s, 6H), 3.65 (s, 6H).

2,3,4,5-四-对-三氟甲基苯基-3' $H$-螺 [环戊二烯并 [2,4]二烯-1,4'-荎] -3'-酮(3c'): 黄色固体, 产率 89\%. m.p. $170 \sim 171{ }^{\circ} \mathrm{C} ;{ }^{1} \mathrm{H}$ NMR (400 MHz, $\left.\mathrm{CDCl}_{3}\right) \delta: 7.43 \sim 7.36$ $(\mathrm{m}, 5 \mathrm{H}), 7.36 \sim 7.32(\mathrm{~m}, 2 \mathrm{H}), 7.31 \sim 7.29(\mathrm{~d}, J=4.0 \mathrm{~Hz}$, $1 \mathrm{H}), 7.28 \sim 7.24(\mathrm{~m}, 1 \mathrm{H}), 7.24 \sim 7.22(\mathrm{~s}, 1 \mathrm{H}), 7.21 \sim 7.18$ (m, 3H). 7.03 (d, J=7.8 Hz, 4H), 6.66 (d, $J=7.8 \mathrm{~Hz}, 4 \mathrm{H})$, $6.16(\mathrm{~d}, J=9.9 \mathrm{~Hz}, 1 \mathrm{H}) .{ }^{13} \mathrm{C} \mathrm{NMR}\left(100 \mathrm{MHz}, \mathrm{CDCl}_{3}\right) \delta$ : $193.8,148.3,146.1,137.2,136.7,135.7,130.7,130.0$, $129.8,129.6,129.3,129.2,128.9,128.8,128.2,126.6$, $126.4,124.9,124.7,122.2,122.1,75.9$. IR (KBr) v: 2999, $1662,1617,1407,1325,1125,1067,1017,849 \mathrm{~cm}^{-1}$. HRMS (ESI) calcld for $\mathrm{C}_{42} \mathrm{H}_{22} \mathrm{~F}_{12} \mathrm{ONa}[\mathrm{M}+\mathrm{Na}]$ 793.1377, found 793.1386.

$2,3,4,5$-四-对-氯苯基-3' $H$ 螺 [环戊二烯并 $[2,4]$ 二 烯-1,4'-菜]-3'-酮 $\left(\mathbf{3 d}^{\prime}\right)^{[13]}$ : 黄色固体, 产率 96\%. m.p. 208 $210{ }^{\circ} \mathrm{C} ;{ }^{1} \mathrm{H}$ NMR (400 MHz, $\left.\mathrm{CDCl}_{3}\right) \delta: 7.39 \sim 7.34$ (m, 2H), $7.33(\mathrm{~s}, 1 \mathrm{H}), 7.30(\mathrm{~d}, J=9.6 \mathrm{~Hz}, 1 \mathrm{H}), 7.25$ (d, $J=$ $7.1 \mathrm{~Hz}, 1 \mathrm{H}), 7.15$ (d, $J=8.5 \mathrm{~Hz}, 4 \mathrm{H}), 6.97$ (d, $J=8.6 \mathrm{~Hz}$, $4 \mathrm{H}), 6.91(\mathrm{~d}, J=8.5 \mathrm{~Hz}, 4 \mathrm{H}), 6.54(\mathrm{~d}, J=8.5 \mathrm{~Hz}, 4 \mathrm{H}), 6.20$ $(\mathrm{d}, J=9.9 \mathrm{~Hz}, 1 \mathrm{H})$.

2,3,4,5-四-3-氟苯基-3' $H$ 螺 [环成二烯并[2,4]二烯1,4'-菜]-3'-酮 $\left(3 \mathbf{e}^{\prime}\right)^{[13]}$ : 黄色固体, 产率 90\%. m.p. 163 $165{ }^{\circ} \mathrm{C} ;{ }^{1} \mathrm{H}$ NMR (400 MHz, $\left.\mathrm{CDCl}_{3}\right) \delta: 7.44 \sim 7.32(\mathrm{~m}$, $4 \mathrm{H}), 7.27$ (s, 1H), 7.15 (dd, $J=14.0,7.9 \mathrm{~Hz}, 2 \mathrm{H}), 7.12 \sim$ $6.93(\mathrm{~m}, 4 \mathrm{H}), 6.81(\mathrm{~d}, J=7.7 \mathrm{~Hz}, 2 \mathrm{H}), 6.77$ (dd, $J=8.4$, $1.8 \mathrm{~Hz}, 2 \mathrm{H}), 6.73(\mathrm{~d}, J=9.8 \mathrm{~Hz}, 2 \mathrm{H}), 6.44(\mathrm{~d}, J=7.8 \mathrm{~Hz}$, $2 \mathrm{H}), 6.32$ (d, $J=10.1 \mathrm{~Hz}, 2 \mathrm{H}), 6.23$ (d, $J=9.9 \mathrm{~Hz}, 1 \mathrm{H})$.

2,3,4,5-四-2-氟苯基-3' $H$ 螺 [环戍二烯并 $[2,4]$ 二烯1,4'-菜]-3'-酮 $\left(3 \mathbf{f}^{\prime}\right)^{[13]}$ : 黄色固体, 产率 92\%. m.p. 239 $240{ }^{\circ} \mathrm{C} ;{ }^{1} \mathrm{H}$ NMR $\left(400 \mathrm{MHz}, \mathrm{CDCl}_{3}\right) \delta: 7.38 \sim 7.34(\mathrm{~m}$, $2 \mathrm{H}), 7.34 \sim 7.30(\mathrm{~m}, 1 \mathrm{H}), 7.28 \sim 2.27(\mathrm{~m}, 5 \mathrm{H}), 7.26 \sim 7.21$ $(\mathrm{m}, 1 \mathrm{H}), 7.18 \sim 7.12(\mathrm{~m}, 3 \mathrm{H}), 6.97(\mathrm{~d}, J=8.4 \mathrm{~Hz}, 3 \mathrm{H})$, $6.91(\mathrm{~d}, J=8.3 \mathrm{~Hz}, 3 \mathrm{H}), 6.55(\mathrm{~d}, J=8.4 \mathrm{~Hz}, 3 \mathrm{H}), 6.24 \sim$ $6.21(\mathrm{~m}, 1 \mathrm{H})$.

2,3,4,5-四丙基-3'H-螺 [环戊二烯并 [2,4]二烯-1,4'-
䓫]-3'-酮 $\left(3 g^{\prime}\right)^{[13]}$ : 黄色固体，产率 76\%. m.p. 188 $189{ }^{\circ} \mathrm{C} ;{ }^{1} \mathrm{H}$ NMR (400 MHz, $\left.\mathrm{CDCl}_{3}\right) \delta: 7.43$ (d, $J=9.9$ $\mathrm{Hz}, 1 \mathrm{H}), 7.25 \sim 7.23(\mathrm{~m}, 1 \mathrm{H}), 7.18 \sim 7.10(\mathrm{~m}, 2 \mathrm{H}), 6.70(\mathrm{~d}$, $J=7.4 \mathrm{~Hz}, 1 \mathrm{H}), 6.22(\mathrm{~d}, J=9.9 \mathrm{~Hz}, 1 \mathrm{H}), 2.24 \sim 2.14(\mathrm{~m}$, $4 \mathrm{H}), 1.96 \sim 1.87(\mathrm{~m}, 2 \mathrm{H}), 1.79 \sim 1.71(\mathrm{~m}, 2 \mathrm{H}), 1.48 \sim 1.40$ $(\mathrm{m}, 4 \mathrm{H}), 0.93 \sim 0.90(\mathrm{~m}, 6 \mathrm{H}), 0.89 \sim 0.83(\mathrm{~m}, 4 \mathrm{H}), 0.62 \sim$ $0.56(\mathrm{~m}, 6 \mathrm{H})$.

2,3,4,5-四(2-噻吩基)-3' $H$ 螺 [环戊二烯并[2,4]二烯1,4'-菜]-3'-酮(3h') ${ }^{[13]}$ : 黄色固体，产率 42\%. m.p. 147 $149{ }^{\circ} \mathrm{C} ;{ }^{1} \mathrm{H}$ NMR (400 MHz, $\left.\mathrm{CDCl}_{3}\right) \delta: 7.44$ (d, $J=9.9$ $\mathrm{Hz}, 1 \mathrm{H}), 7.35 \sim 7.29(\mathrm{~m}, 3 \mathrm{H}), 7.29 \sim 7.25(\mathrm{~s}, 2 \mathrm{H}), 7.22 \sim$ $7.19(\mathrm{~m}, 1 \mathrm{H}), 7.01 \sim 6.96(\mathrm{~m}, 2 \mathrm{H}), 6.95 \sim 6.90(\mathrm{~m}, 2 \mathrm{H})$, $6.91 \sim 6.87(\mathrm{~m}, 2 \mathrm{H}), 6.68 \sim 6.61(\mathrm{~m}, 2 \mathrm{H}), 6.35(\mathrm{dd}, J=3.7$, $1.0 \mathrm{~Hz}, 2 \mathrm{H}), 6.22(\mathrm{~d}, J=9.9 \mathrm{~Hz}, 1 \mathrm{H})$.

2,4-二甲基-3,5-二苯基-3' $H$-螺 [环戊二烯并 $[2,4]$ 二 烯-1,4'-菜]-3'-酮(3i') ${ }^{[13]}$ : 黄色油状物, 产率 $76 \%$. ${ }^{1} \mathrm{H}$ NMR (400 MHz, $\left.\mathrm{CDCl}_{3}\right) \delta: 7.41(\mathrm{~d}, J=9.9 \mathrm{~Hz}, 1 \mathrm{H})$, $7.37 \sim 7.32(\mathrm{~m}, 2 \mathrm{H}), 7.28 \sim 7.27(\mathrm{~m}, 1 \mathrm{H}), 7.25 \sim 7.23(\mathrm{~m}$, $2 \mathrm{H}), 7.23 \sim 7.19(\mathrm{~m}, 2 \mathrm{H}), 7.18(\mathrm{~s}, 1 \mathrm{H}), 7.08 \sim 7.04(\mathrm{~m}$, $2 \mathrm{H}), 7.03 \sim 6.98(\mathrm{~m}, 2 \mathrm{H}), 6.86 \sim 6.81(\mathrm{~m}, 2 \mathrm{H}), 6.22(\mathrm{~d}, J=$ $9.9 \mathrm{~Hz}, 1 \mathrm{H}), 1.96(\mathrm{~s}, 3 \mathrm{H}), 1.46(\mathrm{~s}, 3 \mathrm{H})$.

2,4-二丁基-3,5-二苯基-3'H-螺 [环戊二烯并 $[2,4]$ 二 烯-1,4'-菜]-3'-酮 (3j' $)^{[13]}$ : 黄色固体, 产率 54\%. m.p. $137 \sim 140{ }^{\circ} \mathrm{C} ;{ }^{1} \mathrm{H}$ NMR $\left(400 \mathrm{MHz}, \mathrm{CDCl}_{3}\right) \delta: 7.43 \sim 7.40$ $(\mathrm{m}, 2 \mathrm{H}), 7.39 \sim 7.38(\mathrm{~m}, 1 \mathrm{H}), 7.35 \sim 7.33(\mathrm{~m}, 2 \mathrm{H}), 7.33$ $(\mathrm{m}, 1 \mathrm{H}), 7.32 \sim 7.29(\mathrm{~m}, 1 \mathrm{H}), 7.29 \sim 7.27(\mathrm{~m}, 2 \mathrm{H}), 7.14(\mathrm{~d}$, $J=7.7 \mathrm{~Hz}, 1 \mathrm{H}), 7.11 \sim 7.07(\mathrm{~m}, 3 \mathrm{H}), 6.82 \sim 6.79(\mathrm{~m}, 2 \mathrm{H})$, $6.24(\mathrm{~d}, J=9.9 \mathrm{~Hz}, 1 \mathrm{H}), 2.37 \sim 2.26(\mathrm{~m}, 2 \mathrm{H}), 2.05 \sim 2.00$ $(\mathrm{m}, 1 \mathrm{H}), 1.86 \sim 1.79(\mathrm{~m}, 1 \mathrm{H}), 1.21 \sim 1.13(\mathrm{~m}, 2 \mathrm{H}), 1.11 \sim$ $1.06(\mathrm{~m}, 2 \mathrm{H}), 0.97 \sim 0.87(\mathrm{~m}, 4 \mathrm{H}), 0.66 \sim 0.59(\mathrm{~m}, 3 \mathrm{H})$, $0.55(\mathrm{~m}, 3 \mathrm{H})$.

2,4-二环丙基-3,5-二苯基-3' $H$-螺 [环戊二烯并 [2,4] 二烯-1,4'-菜]-3'-酮 $\left(3 \mathbf{k}^{\prime}\right)^{[13]}$ : 黄色油状物, 产率 $67 \%$. ${ }^{1} \mathrm{H}$ NMR $\left(400 \mathrm{MHz}, \mathrm{CDCl}_{3}\right) \delta: 7.64 \sim 7.60(\mathrm{~m}, 2 \mathrm{H}), 7.56 \sim$ $7.52(\mathrm{~m}, 3 \mathrm{H}), 7.47 \sim 7.46(\mathrm{~m}, 1 \mathrm{H}), 7.45 \sim 7.42(\mathrm{~m}, 3 \mathrm{H})$, $7.25 \sim 7.23(\mathrm{~m}, 1 \mathrm{H}), 7.22 \sim 7.19(\mathrm{~m}, 3 \mathrm{H}), 7.02 \sim 6.97(\mathrm{~m}$, $2 \mathrm{H}), 6.37(\mathrm{~d}, J=9.9 \mathrm{~Hz}, 1 \mathrm{H}), 1.69 \sim 1.65(\mathrm{~m}, 1 \mathrm{H}), 1.36 \sim$ $1.33(\mathrm{~m}, 1 \mathrm{H}), 0.65 \sim 0.59(\mathrm{~m}, 2 \mathrm{H}), 0.43 \sim 0.38(\mathrm{~m}, 2 \mathrm{H})$, $0.29(\mathrm{~m}, 1 \mathrm{H}), 0.26 \sim 0.22(\mathrm{~m}, 1 \mathrm{H}), 0.20 \sim 0.16(\mathrm{~m}, 1 \mathrm{H})$, $0.12 \sim 0.08(\mathrm{~m}, 1 \mathrm{H})$.

辅助材料(Supporting Information) 化合物 $3 b \sim 3 p$ 、 $3 \mathbf{c}^{\prime}$ 的 ${ }^{1} \mathrm{H}$ NMR 和 ${ }^{13} \mathrm{C}$ NMR 原始谱图, 3a、3 $\mathbf{a}^{\prime} 、 3 \mathbf{b}^{\prime}$ 、 $\mathbf{3 d}^{\prime} \sim 3 \mathbf{k}^{\prime}{ }^{1} \mathrm{H}$ NMR 原始谱图. 这些材料可以免费从本刊 网站(http://sioc-journal.cn/)上下载. 


\section{References}

[1] (a) Edrada, R. A.; Stessman, C. C.; Crews, P. J. Nat. Prod. 2003, 66, 939.

(b) Cheng, P.; Ma, Y.; Yao, S.; Zhang, Q.; Wang, E.; Yan, M.; Zhang, X.; Zhang, F.; Chen, J. Bioorg. Med. Chem. Lett. 2007, 17, 5316.

(c) Zhang, Y.; Ge, H.; Zhao, W.; Dong, H.; Xu, Q.; Li, S.; Li, J.; Zhang, J.; Song, Y.; Tan, R. Angew. Chem., Int. Ed. 2008, 47, 5823. (d) Fu, J.; Qin, J.; Zeng, Q.; Huang, Y.; Jin, H.; Zhang, W. Chem. Pharm. Bull. 2010, 58, 1263.

(e) Zhao, J. H.; Wang, Z. Q.; Zhou, Y.; Zhu, G. N.; Yu, C. M. Chin. J. Org. Chem. 2011, 31, 3031 (in Chinese).

(赵金浩, 王宗成, 周勇, 朱国念, 俞传明, 有机化学, 2011, 31, 3031.)

(f) Park, H. B.; Kim, Y.-J.; Lee, J. K.; Lee, K. R.; Kwon, H. C. Org. Lett. 2012, 14, 5002.

(g) Du, W.; Hung, H.; Kuo, P.; Hwang, T.; Shiu, L.; Shiu, K.; Lee, E.; Tai, S.; Wu, T. Org. Lett. 2016, 18, 3042.

(h) Ma, Y.; Fan, C.; Chin. J. Org. Chem. 2016, 36, 2380 (in Chinese).

(马养民, 范超, 有机化学, 2016, 36, 2380.)

[2] Reviews: (a) Roche, S. P.; Porco, Jr., J. Angew. Chem., Int. Ed. 2011, 50, 4068.

(b) Zhuo, C.; Zhang, W.; You, S. Angew. Chem., Int. Ed. 2012, 51, 12662.

(c) Wu, W.-T.; Zhang, L.; You, S.-L. Chem. Soc. Rev. 2016, 45, 1570 .

(d) James, M. J.; O’Brien, P.; Taylor, R. J. K.; Unsworth, W. P. Chem. Eur. J. 2016, 22, 2856.

(e) Nemoto, T.; Hamada, Y. Synlett 2016, 2301.

(f) Wang, H.; Luan, X. Org. Biomol. Chem. 2016, 14, 9451.

(g) Liang, X.-W.; Chen, X.; Zhang, Z.; You S.-L. Chin. Chem. Lett. 2018, 29, 1212.

(h) Wang, K.-K.; Du, W.; Zhu, J.; Chen Y.-C. Chin. Chem. Lett. 2017, 28, 512 .

(i) Wu, W.-T.; Zhang, L. M.; You, S.-L. Acta Chim. Sinica 2017, 75, 419 (in Chinese).

(吴文挺, 张立明, 游书力, 化学学报, 2017, 75, 419.)

[3] (a) Tyman, J. H. P. Synthetic and Natural Phenol; Elsevier: New York, 1996.

(b) Rappoport, Z. The Chemistry of Phenols; John Wiley \& Sons Ltd.: Chichester, 2003.

(c) Weber, M.; Weber, M.; KleineBoymann, M. Phenol. In Ullmann' $s$ Encyclopedia of Industrial Chemistry, Wiley-VCH, Weinheim, 2004.
[4] For selected reviews, see: (a) Kündig, E. P.; Rape, A. Top. Organomet. Chem. 2004, 7, 95.

(b) Quideau, S.; Pouységu, L.; Deffieus, D. Synlett 2008, 467.

(c) Roche, S. P.; Porco, J. A., Jr. Angew. Chem., Int. Ed. 2011, 50, 4068.

(d) Zhuo, C. X.; Zhang, W.; You, S. L. Angew. Chem., Int. Ed. 2012, 51, 12662 .

(e) Lü, W. W.; He, X. C.; Shi, M.; Wang, F. J. Chin. J. Org. Chem. 2019, 39, 532 (in Chinese).

(吕雯雯, 贺信淳, 施敏, 王飞军, 有机化学, 2019, 39, 532.)

(f) Cui, N.; Zhao, Y.; Wang, Y. X. Chin. J. Org. Chem. 2017, 37, 20 (in Chinese).

(崔娜, 赵宇, 王云侠, 有机化学, 2017, 37, 20.)

(g) Lin, S. B.; He, X. R.; Meng, J. P.; Gu, H. N.; Zhang, P. Z.; Wu, J. Chin. J. Org. Chem. 2017, 37, 1864 (in Chinese).

(淮松波，何兴瑞，孟金鹏，顾海宁，张培志，吴军，有机化学， 2017, 37, 1864.)

[5] (a) Roche, S. P.; Porco, J., Jr Angew. Chem., Int. Ed. 2011, 50, 4068 .

(b) Zhuo, C.; Zhang, W.; You, S. Angew. Chem., Int. Ed. 2012, 51, 12662 .

(c) Zhuo, C.; Zheng, C.; You, S. Acc. Chem. Res. 2014, 47, 2558.

(d) Zheng, C.; You, S. Chem. 2016, 1, 830.

(e) Wu, W.; Zhang, L.; You, S. Chem. Soc. Rev. 2016, 45, 1570.

(f) Nemoto, T.; Hamada, Y. Synlett 2016, 27, 2301.

(g) Wang, H.; Luan, X. Org. Biomol. Chem. 2016, 14, 9451.

[6] Nemoto, T.; Ishige, Y.; Yoshida, M.; Kohno, Y.; Kanematsu, M.; Hamada, Y. Org. Lett. 2010, 12, 5020.

[7] Rousseaux, S.; García-Fortanet, J.; Del Aguila Sanchez, M. A.; Buchwald, S. L. J. Am. Chem. Soc. 2011, 133, 9282.

[8] Wu, Q.; Liu, W.; Zhuo, C.; Rong, Z.; Ye, K.; You, S. Angew. Chem., Int. Ed. 2011, 50, 4455.

[9] (a) Nan, J.; Zuo, Z.; Luo, L.; Bai, L.; Zheng, H.; Yuan, Y.; Liu, J.; Luan, X.; Wang, Y. J. Am. Chem. Soc. 2013, 135, 17306. (b) Zuo, Z.; Yang, X.; Liu, J.; Nan, J.; Bai, L.; Wang, Y.; Luan, X. J. Org. Chem. 2015, 80, 3349.

[10] Seoane, A.; Casanova, N.; Quiñones, N.; Mascareñas, J. L.; Gulías, M. J. Am. Chem. Soc. 2014, 136, 7607.

[11] Kujawa, S.; Best, D.; Burns, D. J.; Lam, H. W. Chem. Eur. J. 2014, 20,8599 .

[12] (a) Zheng, J.; Wang, S.; Zheng, C.; You, S. J. Am. Chem. Soc. 2015, 137, 4880. (b) Zheng, C.; Zheng, J.; You, S. ACS Catal. 2016, 6, 262.

[13] Gu, S.; Luo, L.; Liu, J.; Bai, L.; Zheng, H.; Wang, Y.; Luan, X. Org. Lett. 2014, 16, 6132.

[14] Bai, L.; Yuan, Y.; Liu, J.; Wu, J.; Han, L.; Wang, H.; Wang, Y.; Luan, X. Angew. Chem., Int. Ed. 2016, 55, 6946.

(Lu, Y.) 